\title{
Impact of Procedure Time of Preceding Endoscopic Submucosal Dissection on the Difficulty of Laparoscopic Rectal Surgery
}

Hiroaki Nozawa ( $\nabla$ hiroanozawa-gi@umin.ac.jp )

The University of Tokyo https://orcid.org/0000-0002-7891-5986

Kazushige Kawai

The University oof Tokyo

Kazuhito Sasaki

The University of Tokyo

Shigenobu Emoto

The University of Tokyo

Koji Murono

The University of Tokyo

Hirofumi Sonoda

The University of Tokyo

Hiroaki Ishii

The University of Tokyo

Soichiro Ishihara

The University of Tokyo

\section{Research article}

Keywords: Endoscopic submucosal dissection, Procedure time, Rectal cancer, Laparoscopic surgery, Surgical outcomes

Posted Date: July 30th, 2020

DOI: https://doi.org/10.21203/rs.3.rs-44867/v1

License: (c) (i) This work is licensed under a Creative Commons Attribution 4.0 International License. Read Full License

Version of Record: A version of this preprint was published at International Surgery on January 1st, 2021. See the published version at https://doi.org/10.9738/INTSURG-D-20-00030.1. 


\section{Abstract \\ Background}

Endoscopic treatment for gastrointestinal cancer can cause inflammation, edema, and fibrosis formation in the surrounding tissue. Recently, we reported that preceding endoscopic treatment increased the volume of intraoperative blood loss and slightly prolonged the operative time of laparoscopic surgery for rectal cancer. In this study, we addressed which factors, including endoscopic submucosal dissection (ESD)- related parameters, affect the difficulty of laparoscopic rectal surgery.

\section{Methods}

We retrospectively reviewed 24 consecutive patients who underwent ESD followed by laparoscopic surgery for rectal cancer in our hospital. Short-term surgical outcomes were evaluated by intraoperative blood loss and operative time for laparoscopic surgery. The correlations between the surgical outcomes and preoperative parameter were analyzed by scatter diagrams and multiple linear regression analyses.

\section{Results}

The patient cohort comprised 12 men and 12 women. The median distance between primary cancer and anal verge was $7 \mathrm{~cm}$. The median procedure time of ESD was 120 minutes (21 available cases). The scatter diagram graph revealed a positive correlation between the ESD procedure time and estimated blood loss during rectal surgery ( $r=0.26$ ). There was no association between the ESD procedure time and operative time for rectal surgery. Based on multiple linear regression analyses, the ESD procedure time $(p=0.007)$ and tumor location from the anal verge $(p=0.046)$ were independently predictive of intraoperative blood loss. On the other hand, only tumor location was found an independent predictor of surgical time $(p=0.014)$.

\section{Conclusions}

A long session of ESD for rectal cancer may make subsequent laparoscopic surgery difficult based on intraoperative blood loss.

\section{Background}

Endoscopic treatments such as endoscopic mucosal resection (EMR) and endoscopic submucosal dissection (ESD) have been developed as minimally invasive techniques for the en bloc removal of early cancer of the gastrointestinal tract, including the colon and rectum [1-4]. However, some patients with early colorectal cancer (CRC) are referred for additional surgery after endoscopic treatment because of histological findings suggestive of possible metastasis in regional lymph nodes [5]. We recently 
demonstrated that preceding endoscopic treatment increased the volume of intraoperative blood loss and slightly prolonged the operative time forlaparoscopic surgery for early rectal cancer [6].

Electrocoagulation injury to the rectal wall induced by endoscopic treatment may lead to transmural burn causing inflammation of the mesorectum and subsequent fibrosis in the surrounding tissue (a typical case is presented in Fig. 1), which is considered a possible explanation for the results.ESD usually takes longer than EMR $[4,7]$ and is therefore more likely to cause deep mural injury, leading to post-ESD electrocoagulation syndrome [8]. Moreover, a long procedure time was reported to be a risk factor for perforation during colorectal ESD [9]. Collectively we hypothesized that the ESD procedure time is associated with the extent of inflammation, and increases the volume of blood loss and/or operative time during additional laparoscopic rectal surgery.

In this study, we investigated which factors affect the level of difficulty of laparoscopic surgery after ESD for early rectal cancer, particularly focusing on ESD-related parameters.

\section{Methods}

We retrospectively reviewed consecutive patients with early rectal cancer who underwent ESD and subsequent surgery via a laparoscopic approach between November 2012 and February 2020 at the Department of Surgical Oncology, The University of Tokyo Hospital. Patients in whom ESD was discontinued for technical reasons during the procedure were included, whereas patients treated by abdominoperineal resection (APR), those who underwent combined resection of multiple CRCs, and those who needed conversion to open laparotomy were excluded.

All endoscopic procedures were performed by experienced endoscopists in our hospital and affiliated hospitals. The indications and procedure of ESD for rectal lesions were described previously $[2,6]$. Pathological findings of ESD specimens indicating non-curative, namely oncologically insufficient treatment included cancer-positive margin, extensive submucosal invasion ( $\geq 1 \mathrm{~mm} ; \mathrm{pT} 1 \mathrm{~b}$ [10]), lymphatic and venous infiltration, dedifferentiated component, and grade 2 or 3 budding based on the Japanese Society for Cancer of the Colon and Rectum guidelines [5].

All surgical procedures were performed as described previously [6]. Briefly, low tie of the inferior mesenteric artery was performed preserving the left colic artery via the medial-to-lateral approach. The splenic flexure was taken down when required. Then, sharp dissection in front of the prehypogastric nerve fascia and behind Denonvilliers' fascia was performed based on total mesorectal excision. In anterior resection, a defunctioning stoma was created at the surgeon's discretion considering the patient's comorbidities and risk of anastomotic leakage, such as an incomplete doughnut after stapled anastomosis and positive findings on air insufflation. A diverting stoma was created in all patients undergoing intersphicteric resection.

As baseline variables, age, sex, body mass index (BMI), ESD procedure time, interval between ESD and surgery, tumor location from the anal verge, tumor size, serum hemoglobin level, prothrombin timeinternational normalized ratio (PT-INR), and activated partial thromboplastin time (APTT) before surgery 
were retrieved from medical charts. The normal ranges of PT-INR and APTT are $0.85-1.15$ and 25.5-36.1 seconds, respectively, in our hospital. The main outcomes of interest in this study were operative time and estimated volume of blood loss during laparoscopic surgery. Other clinical and pathological outcomes, such as perioperative blood transfusion, post-operative complications graded according to the ClavienDindo classification [11], postoperative hospital stay, pathological depth (pT), and lymph node metastasis $(\mathrm{pN})$, were reviewed.

To examine relationships between numerical variables, Spearman's rank correlation coefficient (rs) was calculated. To investigate associations of preoperative variables with the surgical outcomes, multivariate analysis was performed using a multiple linear regression model with a stepwise (forward selection/backward elimination) method (significance level to enter $=0.25$, significance level to stay $=$ 0.1). All statistical analyses were conducted using the JMP 15.1 software (SAS Institute Inc., Cary, NC, USA). A $p$ value below 0.05 was considered to indicate significance.

\section{Results}

Twenty-eight patients underwent salvage surgery via a laparoscopic approach after ESD in the study period. As two patients underwent APR, one patient had synchronous sigmoid colon cancer, and one patient needed conversion to open laparotomy, 24 patients were analyzed in the current study (Fig. 2).

The characteristics of subjects ( 12 men and 12 women, median age: 63 years) are summarized in Table 1. The ESD procedure time was available for 21 patients, which ranged 28 to 720 minutes with a median of 120 minutes. The primary tumor was located $7 \mathrm{~cm}$ (median) from the anal verge. Two patients had chronic anti-thrombotic therapies that were stopped before surgery. The preoperative PT-INR and APTT were normal in all patients. Additional surgery was performed a median of 62 days after ESD. 
Table 1

Characteristics of the study patients $(n=24)$

\section{Characteristics}

\begin{tabular}{|lll|}
\hline Age, years & Median (range) & $63(44-85)$ \\
\hline Sex & Male & $12(50 \%)$ \\
\hline Hemoglobin, g/dL & Median (range) & $23.8(16.0-30.4)$ \\
\hline ESD procedure time, min a & Median (range) & $13.4(10.2-15.8)$ \\
\hline Interval between ESD and surgery, days & Median (range) & $120(28-720)$ \\
\hline Distance from AV, cm & Median (range) & $62(31-190)$ \\
\hline Tumor size, mm & Median (range) & $7(1-15)$ \\
\hline PT-INR & Median (range) & $22(7-110)$ \\
\hline $\begin{array}{l}\text { APTT } \\
\text { ESD endoscopic submucosal dissection, AV anal verge, PT-INR prothrombin time-international } \\
\text { normalized ratio, APTT activated partial thromboplastin time }\end{array}$ & Normal & $0(0 \%)$ \\
\hline a unavailable data in three patients were excluded & Prolonged & $24(100 \%)$ \\
\hline
\end{tabular}

The intraoperative and postoperative outcomes are summarized in Table 2. The median blood loss was $75 \mathrm{~mL}$ and the median surgical time was 341 minutes. A stoma was created in 16 patients. Seven patients developed grade 2 or higher post-operative complications that were treated conservatively except for leg compartment syndrome. There was no perforation in the rectal wall during surgery and no anastomotic leakage. Patients were discharged around two weeks after surgery. Two patients in whom ESD was discontinued and one with a positive vertical margin were diagnosed with pT2 tumors. Regional lymph node metastases were detected in five patients (21\%). During the follow-up period (median: 34 months), only one patient developed lung metastases after rectal surgery, which were removed by videoassisted thoracoscopic surgery. There were no deaths. 
Table 2

Intraoperative and postoperative outcomes $(n=24)$

\begin{tabular}{|lll|}
\hline Characteristics & & Value \\
\hline Estimated blood loss, $\mathrm{mL}$ & Median (range) & $75(0-490)$ \\
\hline Operative time, min & Median (range) & $341(174-449)$ \\
\hline Stoma creation & & $16(67 \%)$ \\
\hline Blood transfusion & & $0(0 \%)$ \\
\hline Morbidity, grade 2- & & $7(29 \%)$ \\
\hline & Anastomotic bleeding & 1 \\
\hline & Small bowel obstruction & $1^{\mathrm{a}}$ \\
\hline Recurrence, organ & Pelvic abscess & 1 \\
\hline a Occurred in an identical patient & Wound infection & 1 \\
\hline pN & Urinary tract infection & 1 \\
\hline Hospital stay after surgery, days & Renal dysfunction & 1 \\
\hline MT & Leg compartment syndrome & $1^{\mathrm{a}}$ \\
\hline & pT1 & 1 \\
\hline & pT2 & $17(10-43)$ \\
\hline & pNo & $21(87 \%)$ \\
\hline & pN1 & $3(13 \%)$ \\
\hline
\end{tabular}


Table 3

Stepwise linear regression analyses of preoperative

factors for surgical outcomesa) Blood loss, $\mathrm{mL}$

\begin{tabular}{|lll|}
\hline Variable & Estimate & $\mathrm{p}$ value \\
\hline Intercept & 130.55 & 0.023 \\
\hline ESD procedure time, min & 0.41 & 0.007 \\
\hline Distance from AV, cm & -12.66 & 0.046 \\
\hline Model utility test: $\mathrm{p}=0.0017$ & & \\
ESD endoscopic submucosal dissection, $A V$ anal verge \\
Variable & Estimate & $\mathbf{p}$ value \\
\hline Intercept & 383.09 & $<0.0001$ \\
\hline Distance from AV, cm & -10.14 & 0.014 \\
\hline Model utility test: $\mathrm{p}=0.092$ & & \\
AV anal verge & & \\
\hline b) Operative time of laparoscopic surgery, min \\
\hline
\end{tabular}

We performed scatter diagram analyses to assess correlations between ESD-related parameters and short-term surgical outcomes. As shown in Fig. 3, there was a weak positive correlation between the ESD procedure time and estimated blood loss in laparoscopic rectal surgery ( $r=0.26)$. In contrast, there was no association between the ESD procedure time and operative time for rectal surgery $(r s=0.15)$. The interval from ESD to laparoscopic surgery was not correlated with intraoperative blood loss $(r s=0.11)$ or operative time for rectal surgery $(r s=0.00)$.

In order to address relationships between clinicopathological factors and surgical outcomes, multiple linear regression analyses were performed. Based on stepwise linear regression analysis, the ESD procedure time and tumor location from the anal verge (estimate: $0.41 \mathrm{~mL} / \mathrm{min}, \mathrm{p}=0.007$, and estimate: $-12.66 \mathrm{~mL} / \mathrm{cm}, \mathrm{p}=0.046$, respectively) were independent predictors for intraoperative blood loss (Table 3A), whereas age, sex, body mass index, hemoglobin level, and treatment interval were not. By a similar analysis, the optimal model to predict surgical time included only tumor location (estimate: $-10.14 \mathrm{~min} / \mathrm{cm}, \mathrm{p}=0.014$, Table $3 \mathrm{~B}$ ). In these analyses, it was confirmed that the residuals followed a normal distribution (data not shown).

\section{Discussion}

Our recent study shed light on the adverse effects of preceding endoscopic therapies on laparoscopic rectal surgery in terms of intraoperative bleeding [6]. In that study, the median volume of blood loss was larger in patients treated by ESD than by EMR $(80 \mathrm{~mL}$ vs $20 \mathrm{~mL})$ [6], suggesting a significant impact of 
cautery duration during endoscopic treatment on the extent of inflammation in the mesorectum and beyond. These findings were an impetus to the current study, and we found a positive correlation between the ESD procedure time and estimated volume of blood loss during laparoscopic rectal surgery.

The ESD procedure time reflects its technical difficulty. In general, the ESD procedure time increases according to tumor size. In a study of specialized hospitals in Japan, the average ESD procedure time was 129 minutes for tumors $\geq 40 \mathrm{~mm}$ in size whereas it was 66 minutes for tumors of 20-29 mm [12]. In addition, a long ESD procedure time is often required for tumors with severe submucosal fibrosis [13], which may be associated with massive invasion into the submucosa or other histological characteristics, e.g. desmoplastic reaction [14]. The median ESD procedure time for our patients who were referred for salvage surgery (120 minutes) was longer than that reported by a systematic review (75 minutes), although it ranged widely in both our cohort and the review (28-720 minutes and 5-600 minutes, respectively) [15]. We consider it important to not only distinguish tumors with out-of-indication features from those of Tis or those invading the submucosal layer less than $1 \mathrm{~mm}$ as pre-ESD diagnosis, but also to pay attention to findings of possible massive submucosal invasion, such as muscle-retracting sign during ESD, considering the correlation between a long session of ESD and the difficulty of subsequent laparoscopic rectal surgery demonstrated in the current study.

After neovascularization that occurs as a pathological response to inflammation, fibrosis generally develops at a delayed stage in the healing process [16]. Thus, we initially hypothesized that the long interval between ESD and surgery will affect short-term surgical outcomes. However, we observed no significant effects of the treatment interval on intraoperative blood loss or surgical time. This suggested that the timing of salvage surgery does not matter in terms of the difficulty of laparoscopic rectal surgery although our findings may contain type II errors.

In addition to the ESD procedure time, the distance between the tumor and the anal verge was significantly associated with increased blood loss during laparoscopic rectal surgery after ESD in the current study. It was also correlated with a longer time for laparoscopic rectal surgery. Previous studies in tertiary hospitals also reported that a low tumor location is associated with difficult laparoscopic surgery when evaluated by these outcome measures in rectal cancer patients who did not undergo preceding ESD $[17,18]$.

We recognize that the current study has several limitations. First, this was a retrospective study conducted at a single hospital with a limited number of surgical patients. Another important limitation of the study is that different endoscopists performed ESD; laparoscopic surgery was also performed by many doctors, although they formed board-certified expert surgeon teams. Moreover, we were unable to assess the extent of mesorectal inflammation in other patients who received ESD for rectal cancer without additional surgery. We did not evaluate the severity of inflammation in the surgically resected specimens histologically because regional lymph nodes were removed from the mesorectum as routine procedure for pathological examination in Japan. Lastly, the findings obtained from Japanese patients 
may not necessarily reflect those in patients in western countries because of differences in patient background, e.g. BMI distribution.

\section{Conclusions}

The current study revealed that the procedure time of preceding ESD for rectal cancer is associated with increased blood loss, one of the surrogate markers of the difficulty of laparoscopic surgery. Doctors should be aware of the impact of ESD on salvage surgery when ESD results in non-curative treatment. It is therefore important to communicate well among endoscopists, surgeons, and pathologists before initiating treatment, and to accurately diagnose the tumor depth even during ESD.

\section{Abbreviations}

APR

abdominoperineal resection; APTT:activated partial thromboplastin time; AV:anal verge; BMI:body mass index; CRC:colorectal cancer; EMR:endoscopic mucosal resection ; ESD:endoscopic submucosal dissection; PT-INR:prothrombin time-international normalized ratio.

\section{Declarations}

\section{Ethics approval:}

This study was performed in line with the principles of the Declaration of Helsinki. Approval was granted by the Ethics Committee of The University of Tokyo (\#3252-10).

\section{Consent to participate}

Informed consent was obtained from all individual participants included in the study.

\section{Consent for publication:}

not applicable

\section{Data availability}

The datasets generated during and/or analysed during the current study are not publicly available because they are derived from the patient database of the hospital and hence subject to confidentiality, but are available from the corresponding author on reasonable request.

\section{Competing Interests:}

The authors declare that they have no competing interests. 


\section{Funding}

This work was supported by Grants-in-Aid for Scientific Research (C: grant number; 18K07194, C: grant number; 19K09114, and C : grant number; 19K09115) from the Japan Society for the Promotion of Science. The funders had no role in the study design, data collection and analysis, decision to publish, or preparation of the manuscript.

\section{Authors' Contributions}

$\mathrm{HN}$ and SI contributed to the study conception and design. Material preparation, data collection and analysis were performed by KK, KS, SE, KM, HS, and HI. All authors contributed to the treatment of patients. The first draft of the manuscript was written by $\mathrm{HN}$, and all authors commented on previous versions of the manuscript. All authors read and approved the final manuscript.

\section{References}

1. Tanaka S, Oka S, Kaneko I, et al. Endoscopic submucosal dissection for colorectal neoplasia: possibility of standardization. Gastrointest Endosc. 2007;66:100-7.

2. Niimi K, Fujishiro M, Kodashima S, et al. Long-term outcomes of endoscopic submucosal dissection for colorectal epithelial neoplasms. Endoscopy. 2010;42:723-9.

3. Saito Y, Uraoka T, Yamaguchi Y, et al. A prospective, multicenter study of 1111 colorectal endoscopic submucosal dissections (with video). Gastrointest Endosc. 2010;72:1217-25.

4. Tajika M, Niwa Y, Bhatia V, et al. Comparison of endoscopic submucosal dissection and endoscopic mucosal resection for large colorectal tumors. Eur J Gastroenterol Hepatol. 2011;23:1042-9.

5. Hashiguchi Y, Muro K, Saito Y, et al. Japanese Society for Cancer of the Colon and Rectum (JSCCR) guidelines 2019 for the treatment of colorectal cancer. Int J Clin Oncol. 2020;25:1-42.

6. Nozawa $\mathrm{H}$, Ishii $\mathrm{H}$, Sonoda $\mathrm{H}$, et al. Effects of preceding endoscopic treatment on laparoscopic surgery for early rectal cancer. Colorectal Dis. 2020. doi:10.1111/codi.14989.

7. Arezzo A, Passera R, Marchese N, et al. Systematic review and meta-analysis of endoscopic submucosal dissection vs endoscopic mucosal resection for colorectal lesions. United European Gastroenterol J. 2016;4:18-29.

8. Yamashina T, Takeuchi Y, Uedo N, et al. Features of electrocoagulation syndrome after endoscopic submucosal dissection for colorectal neoplasm. J Gastroenterol Hepatol. 2016;31:615-20.

9. Yamamoto K, Shimoda R, Ogata S, et al. Perforation and Postoperative Bleeding Associated with Endoscopic Submucosal Dissection in Colorectal Tumors: An Analysis of 398 Lesions Treated in Saga, Japan. Intern Med. 2018;57:2115-22.

10. Japanese Society for Cancer of the Colon and Rectum. Japanese Classification of Colorectal, Appendiceal, and Anal Carcinoma: the 3d English Edition. [Secondary Publication] J Anus Rectum 
Colon. 2019;3:175-95.

11. Dindo D, Demartines N, Clavien PA. Classification of surgical complications: a new proposal with evaluation in a cohort of 6336 patients and results of a survey. Ann Surg. 2004;240:205-13.

12. Saito Y, Yamada M, So E, et al. Colorectal endoscopic submucosal dissection: Technical advantages compared to endoscopic mucosal resection and minimally invasive surgery. Dig Endosc. 2014;26:52-61.

13. Kaosombatwattana U, Yamamura T, Limsrivilai J, et al. Preoperative endoscopic predictors of severe submucosal fibrosis in colorectal tumors undergoing endoscopic submucosal dissection. Endosc Int Open. 2019;7:E421-30.

14. Kim EK, Han DS, Ro Y, et al. The submucosal fibrosis: what does it mean for colorectal endoscopic submucosal dissection? Intest Res. 2016;14:358-64.

15. Akintoye E, Kumar N, Aihara H, et al. Colorectal Endoscopic Submucosal Dissection: A Systematic Review and Meta-Analysis. Endosc Int Open. 2016;4:E1030-44.

16. Diegelmann RF, Evans MC. Wound healing: an overview of acute, fibrotic and delayed healing. Front Biosci. 2004;9:283-9.

17. Akiyoshi T, Kuroyanagi $\mathrm{H}$, Oya M, et al. Factors affecting the difficulty of laparoscopic total mesorectal excision with double stapling technique anastomosis for low rectal cancer. Surgery. 2009;146:483-9.

18. Ogiso S, Yamaguchi T, Hata $\mathrm{H}$, et al. Evaluation of factors affecting the difficulty of laparoscopic anterior resection for rectal cancer: "narrow pelvis" is not a contraindication. Surg Endosc. 2011;25:1907-12.

\section{Figures}




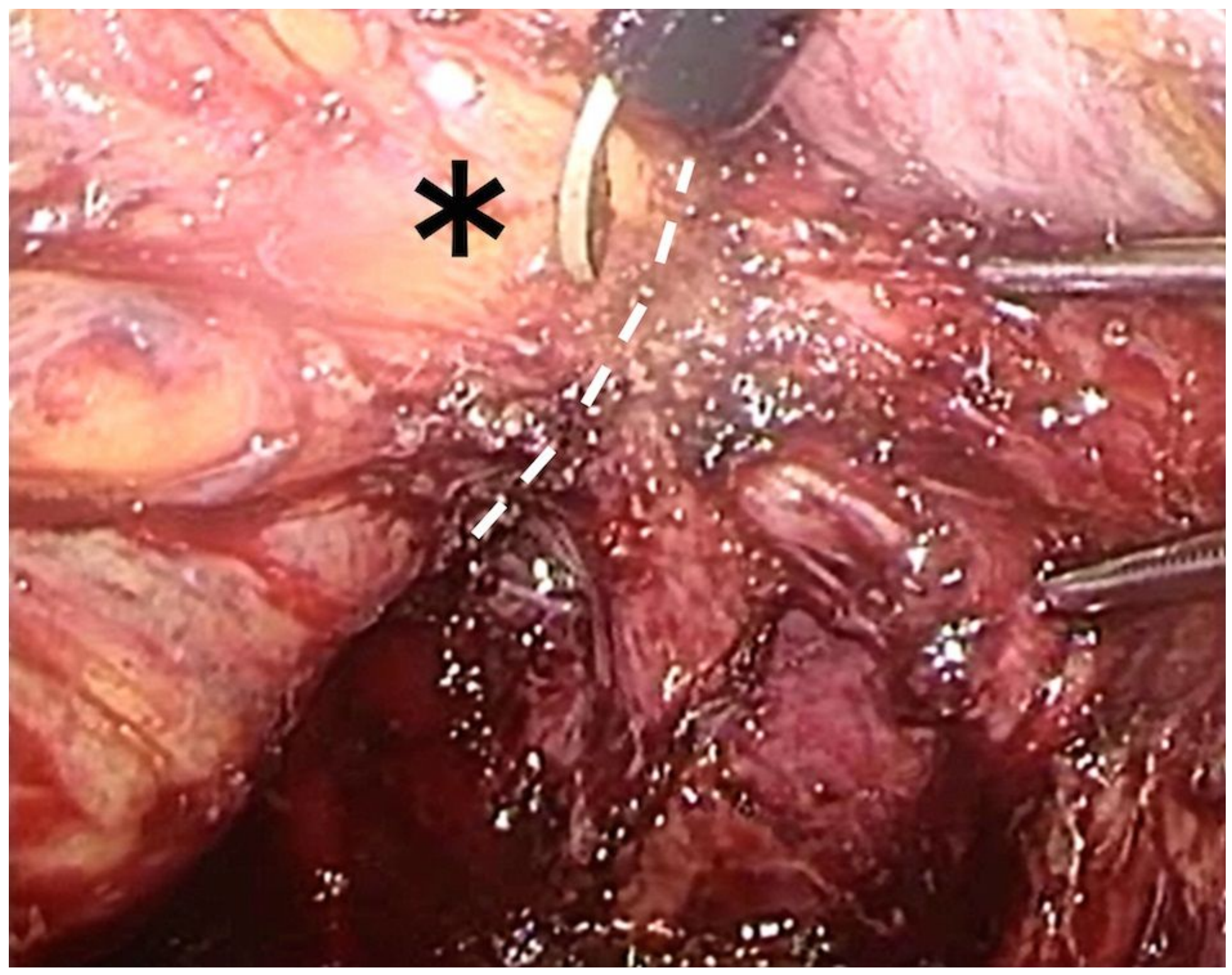

Figure 1

An operative view of severe fibrosis in the dissection plane in laparoscopic rectal surgery after ESD. An imaginary dissection line was drawn as a white dashed line. *: mesorectum. 


\section{Patients who underwent laparoscopic rectal surgery after preceding ESD for rectal cancer between Nov 2012 and Feb 2020 (n=28)}

\section{Excluded;}

Abdominoperineal resection $(n=2)$

Combined resection of synchronous sigmoid colon cancer $(n=1)$ Conversion to laparotomy $(n=1)$

Patients subjected to the analyses $(n=24)$

Figure 2

Flow chart of patient selection. 


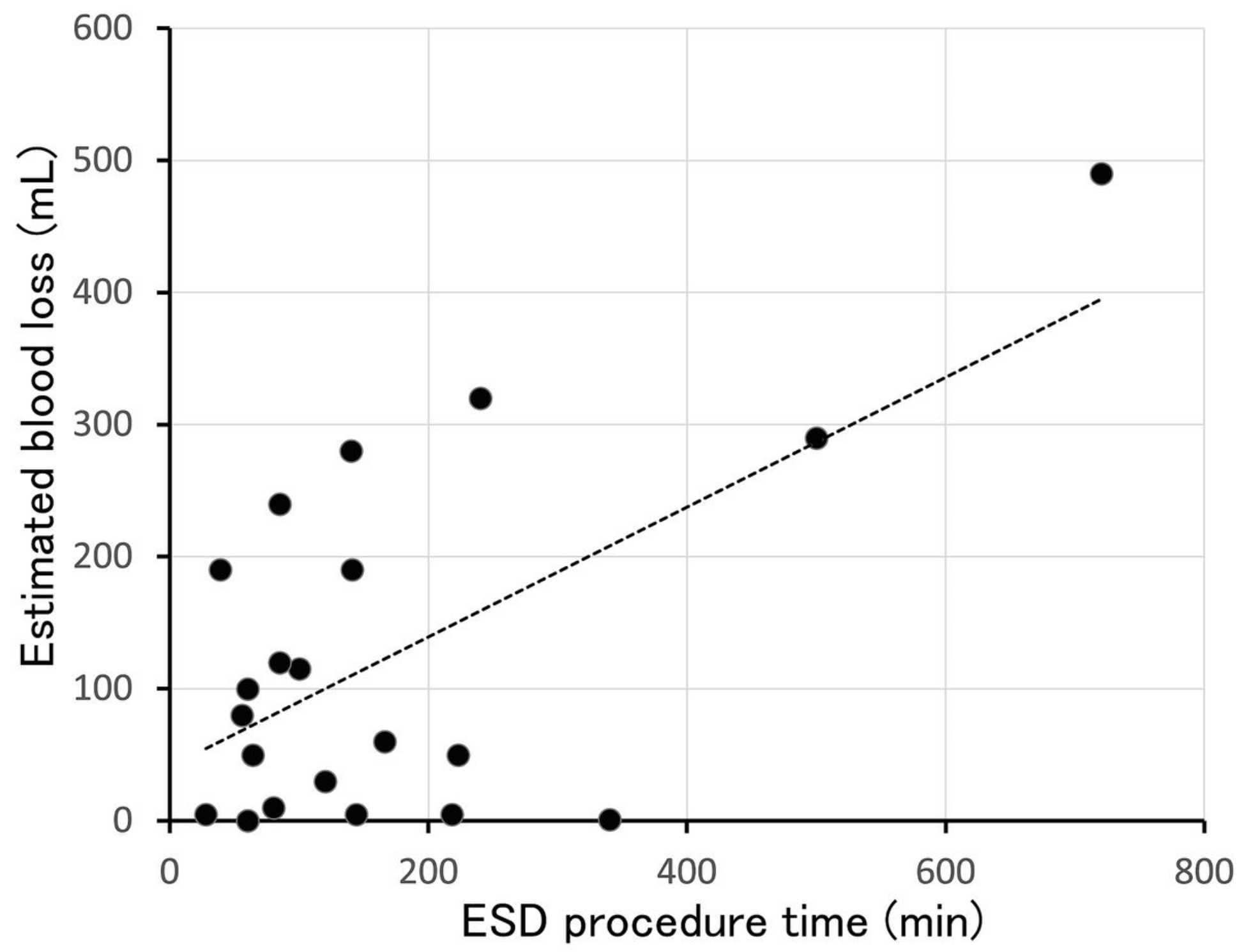

Figure 3

Scatter plot figure for correlation analyses of the ESD procedure time and intraoperative blood loss $(n=21)$. Spearman's correlation coefficient was 0.26 . 